\title{
Metabolism of tellurium, antimony and germanium simultaneously administered to rats
}

\author{
Akihiro Kobayashi and Yasumitsu Ograa \\ Graduate School of Pharmaceutical Sciences, Chiba University, 1-8-1 Inohana, Chuo, Chiba 260-8675, Japan \\ aPresent address: Laboratory of Chemical Toxicology and Environmental Health, Showa Pharmaceutical University \\ 3-3165 Higashi-Tamagawagakuen, Machida, Tokyo 194-8543, Japan
}

(Received March 6, 2009 ; Accepted March 24, 2009)

\begin{abstract}
Recently, tellurium (Te), antimony ( $\mathrm{Sb}$ ) and germanium $(\mathrm{Ge})$ have been used as an alloy in phase-change optical magnetic disks, such as digital versatile disk-random access memory (DVDRAM) and DVD-recordable disk (DVD-RW). Although these metalloids, the so-called "exotic" elements, are known to be non-essential and harmful, little is known about their toxic effects and metabolism. Metalloid compounds, tellurite, antimonite and germanium dioxide, were simultaneously administered to rats. Their distributions metabolites were determined and identified by speciation. Te and $\mathrm{Sb}$ accumulated in red blood cells (RBCs): Te accumulated in RBCs in the dimethylated form, while Sb accumulated in the inorganic/non-methylated form. In addition, trimethyltelluronium (TMTe) was the urinary metabolite of $\mathrm{Te}$, whereas $\mathrm{Sb}$ in urine was not methylated but oxidized. Ge was also not methylated in rats. These results suggest that each metalloid is metabolized via a unique pathway.
\end{abstract}

Key words: Tellurium, Antimony, Germanium, ICP-MS, Speciation, Hemoglobin

\section{INTRODUCTION}

Metalloids possess properties that are intermediate between those of metals and non-metals. They are widely used in industry because of their unique chemical and physical properties. Recently, tellurium (Te), antimony $(\mathrm{Sb})$, and germanium $(\mathrm{Ge})$ have been used as an alloy in phase-change optical magnetic disks, such as digital versatile disk-random access memory (DVD-RAM) and DVD-recordable disk (DVD-RW) (Yamada et al., 2002). Since the recycle of these metalloids from the disks has not been established yet, environmental contamination of the metalloids is really feared, and exposure to these metalloids is expected to increase in everyday life. However, although these metalloids, the so-called "exotic" elements, are known to be non-essential and harmful, little is known about their toxic effects and metabolism. Some clinical manifestations of Te toxicosis are known: nausea, vomiting, garlic breath and garlic odor in sweat are commonly found in Te toxicosis patients (Taylor, 1996). We have reported that the major urinary Te metabolite is trimethyltelluronium (TMTe) and that Te accumulated in red blood cells (RBCs) as a dimethylated Te compound in rats (Ogra et al., 2008). Together, those results suggest that the main route of Te metabolism is methylation. In the case of $\mathrm{Sb}$ toxicosis, the clinical manifestations are nausea, vomiting, diarrhea, hematuria, and nephrotoxicity. In addition, the incidence of lung cancer has increased in industry workers exposed to $\mathrm{Sb}$ (McCallum, 2005). It is reported that $\mathrm{Sb}$ accumulated in RBCs of rats administered antimonite, although the chemical form of accumulated $\mathrm{Sb}$ is still unclear (Poon et al., 1998). It is also reported that antimonate and trimethylantimony chloride were detected in urine of workers exposed to $\mathrm{Sb}$ (Krachler and Emons, 2001). On the other hand, Ge toxicity is less known compared to the above two metalloids. There are reports of renal failure and deafness as the clinical manifestations of Ge toxicosis (Vanholder et al., 2002; Yamasoba et al., 2007). However, Ge metabolism and its metabolites remain unknown. Although these metalloids are actually environmental toxicants, drugs containing them are currently in use or being developed because of their unique toxicological effects. Ammonium trichloro (dioxoethylene-o, $\mathrm{o}^{\prime}$ ) tellurate (AS101) is a Te-containing drug that

Correspondence: Yasumitsu Ogra (E-mail: ogra@ac.shoyaku.ac.jp) 
functions as an antitumor agent and an immunomodulator (Frei et al., 2008). Meglumine antimonate and sodium stibogluconate are the drugs of first choice for leishmaniasis (Santos et al., 2008). In addition, an Sb compound was developed as an antitumor agent (Sharma et al., 2008). A Ge-containing drug, propagermanium, was evaluated for potential use as an immunomodulator (Hirayama et al., 2003). As mentioned above, as the risk of exposure to $\mathrm{Te}, \mathrm{Sb}$ and $\mathrm{Ge}$ is expected to increase, the toxicological effects of these metalloids should be fully understood.

As non-metallic elements, metalloids are utilized in the metabolic pathways of animals to form organometallic compounds having carbon-metalloid covalent bond(s). Therefore, it is necessary to identify the organometallic compounds to clarify the metabolic pathways of metalloids and to understand the toxicological or pharmacological effects of the metalloids. Speciation is one of the most commonly employed analytical techniques for the separation and detection of metal/metalloid-containing species in biological samples. A hyphenated technique that combines HPLC with an inductively coupled plasma mass spectrometer (ICP-MS) is a powerful tool for the speciation of metal/metalloid-containing biomolecules (Haraguchi, 2004; Szpunar, 2005).

In the present study, we elucidated the metabolism of $\mathrm{Te}, \mathrm{Sb}$ and $\mathrm{Ge}$ in rats simultaneously administered with sodium tellurite, antimony trioxide, germanium dioxide. In particular, we determined the concentrations of Te, $\mathrm{Sb}$ and $\mathrm{Ge}$ in tissues and excreta, and performed speciation of the metalloids in extracellular fluid, such as urine and blood. In the case of arsenic (As) metabolism, As is transformed by such enzymes as reductase (glutathione S-transferase omega) and methyltransferase (arsenic methyl transferase-3 or Cyt19) (Hayakawa et al., 2005; Naranmandura et al., 2006). Since Sb belongs to the same group as As, it is expected to share the same metabolic pathway with As. Indeed, methylation is an alternate metabolic pathway of selenium (Se) in addition to glycosylation (Ogra et al., 2002). In our previous study, we found that Te was also metabolized by methyltransferase, suggesting that Te partly shares the same metabolic pathway with Se (Ogra et al., 2008). Thus, we also evaluated whether these enzymes were induced or not by repeated administration of the metalloids.

\section{MATERIALS AND METHODS}

\section{Chemicals}

Sodium tellurite, antimony trioxide, germanium dioxide, and dimethylarsenic acid (cacodylic acid, DMA (V)) were purchased from Wako Pure Chemical Industries Ltd.
(Osaka, Japan). All reagents were of analytical grade or the highest grade available. Purified water $(18.3 \mathrm{M} \Omega / \mathrm{cm})$ from Milli-Q SP (Millipore, Bedford, MA, USA) was used throughout. Trizma Base and $\mathrm{HCl}$ were purchased from Sigma-Aldrich Co. (St. Louis, MO, USA). Te, Sb and Ge standard solutions $(1,000 \mu \mathrm{g} / \mathrm{ml})$ for ICP-MS were purchased from Wako Pure Chemical Industries Ltd..

\section{Animal experiments}

All animal experiments were carried out according to the "Principles of Laboratory Animal Care" (NIH version, revised, 1996) and the Guidelines of the Animal Investigation Committee, Graduate School of Pharmaceutical Sciences, Chiba University, Japan.

\section{Single oral administration}

Five-week-old male Wistar rats were purchased from Japan Clea Inc. (Tokyo, Japan). Three rats were maintained in each stainless steel wire cage at $22 \pm 2^{\circ} \mathrm{C}$ with a light/dark cycle of $12 \mathrm{hr} / 12 \mathrm{hr}$, and fed a commercial diet (MF, Oriental Yeast Co. Ltd., Tokyo, Japan) and tap water ad libitum. After acclimation for 7 days, three rats per group were housed individually in a plastic metabolic cage to collect urine and feces over a 24-hr period until sacrifice. The rats were perorally administered Te, Sb and Ge each at a dose of $2.0 \mu \mathrm{mol} / \mathrm{kg}$ body weight. Sodium tellurite and germanium dioxide were dissolved in diluted sodium hydroxide solution, and antimony trioxide was dissolved in diluted hydrochloric acid. Two kinds of solution were administered one by one at $5 \mathrm{ml} / \mathrm{kg}$ body weight. Sodium tellurite, antimony trioxide or germanium dioxide is one of the stable chemical forms of each metalloid in the environment. Thus, the chemical forms were ingested in the study. The dose of metalloids was decided on the basis of our previous results (Ogra et al., 2008). Forty-eight hr after administration, the animals were sacrificed. Blood was collected under light ether anesthesia into plain and heparin-coated tubes, and clotted blood in the plain tube was centrifuged at $1,600 \mathrm{x} \mathrm{g}$ for $10 \mathrm{~min}$ to obtain serum. Then, the liver, kidneys, lungs, testes, spleen and brain were excised. Blood cells in the heparincoated tube were washed three times with Tris-buffered saline, and buffy coat was removed to prepare RBCs. RBCs were punctured in $10 \mathrm{mmol} / 1$ Tris- $\mathrm{NO}_{3}, \mathrm{pH} 7.4$, and centrifuged at $15,000 \mathrm{x} \mathrm{g}$ for $30 \mathrm{~min}$ to obtain $\mathrm{RBC}$ lysate.

\section{Repeated oral administration}

After acclimation for 7 days under the same conditions as those mentioned above, three rats per group were housed individually in a plastic metabolic cage to collect 
urine and feces over a 12-hr period from $12 \mathrm{hr}$ before the first administration until sacrifice. The rats were perorally administered $\mathrm{Te}, \mathrm{Sb}$ and $\mathrm{Ge}$ each at a dose of $2.0 \mu \mathrm{mol} / \mathrm{kg}$ body weight three times at days 0,4 and 8 . Sodium tellurite and germanium dioxide were dissolved in diluted sodium hydroxide solution, and antimony trioxide was dissolved in diluted hydrochloric acid. Two kinds of solution were administered one by one at $5 \mathrm{ml} / \mathrm{kg}$ body weight. Twenty days after the first administration, the animals were sacrificed. Blood was collected under light ether anesthesia and clotted blood was centrifuged at 1,600 x g for $10 \mathrm{~min}$ to obtain serum. Then, the liver, kidneys, lung, testes, spleen and brain were excised.

\section{Determination of metalloid concentration in samples}

The concentrations of $\mathrm{Te}, \mathrm{Sb}$ and $\mathrm{Ge}$ in individual organs, blood, urine and feces were determined with an ICP-MS (HP4500, Agilent Technologies, Tokyo, Japan). Mass calibration and parameter optimization for ICP-MS were performed daily using a tuning solution containing $10 \mathrm{ng} / \mathrm{ml}$ each of lithium, yttrium, cerium and thallium (Agilent Technologies) prior to use. Te, Sb and Ge were monitored at m/z 125, 121 and 72, respectively.

\section{Speciation of metalloids in urine and oxidized RBC lysate by HPLC-ICP-MS}

The HPLC system consisted of an on-line degasser (DG660B-2, GL Science, Tokyo, Japan), an HPLC pump (PU610, GL Science), a six-port injector (model 7125, Rheodyne, CA, USA) with $20 \mu 1$ sample loops, and a column. A multi-mode gel filtration column, Shodex ${ }^{\circledR}$ Asahipak GS-220HQ (7.5 i.d. $\times 300 \mathrm{~mm}$, with a guard column, 7.5 i.d. $\times 75 \mathrm{~mm}$, Showa Denko, Tokyo, Japan), was injected with a $20 \mu \mathrm{l}$ aliquot of the urine or oxidized RBC lysate sample, and was eluted with $50 \mathrm{mmol} / 1$ ammonium acetate, $\mathrm{pH} 6.5$, at a flow rate of $0.6 \mathrm{ml} / \mathrm{min}$. The eluate was introduced directly into the Babington nebulizer of the ICP-MS, and Te, $\mathrm{Sb}$ and Ge were monitored at $\mathrm{m} / \mathrm{z}$ 125, 121 and 72, respectively.

\section{Speciation of metalloids in RBC lysate by HPLC-PDA-ICP-MS}

The HPLC system (Prominence, Shimadzu, Kyoto, Japan) equipped with a photodiode array detector between the HPLC and the ICP-MS was used to monitor the elution of proteins. A gel filtration column, Shodex ${ }^{\circledR}$ KW803 (8.0 i.d. $\times 300$ mm, Showa Denko), was injected with a $200 \mu \mathrm{l}$ aliquot of the RBC lysate, and was eluted with $50 \mathrm{mmol} / 1$ Tris- $\mathrm{NO}_{3}, \mathrm{pH} 7.4$, at a flow rate of $0.6 \mathrm{ml} /$ min. The eluate was introduced into the photodiode array detector to monitor the elution of heme-containing protein at 280 and $550 \mathrm{~nm}$, and then introduced into the Babington nebulizer of the ICP-MS to monitor Fe, Ge, As, $\mathrm{Se}, \mathrm{Sb}$ and $\mathrm{Te}$ at $\mathrm{m} / \mathrm{z}$ 57, 72, 75, 82, 121 and 125, respectively.

\section{RESULTS AND DISCUSSION}

\section{Distribution of metalloids to organs and blood cells}

Te was distributed predominantly to blood and slightly to serum by single and repeated oral administrations of the three metalloids (Figs. 1A and B). This suggests that Te exists in blood cells in bloodstream. Our previous study demonstrated that Te solely administered distributed predominantly to RBCs (Ogra et al., 2008). The amount of accumulated Te in RBCs of singly administered rats was comparable to that of repeatedly administered rats, suggesting that accumulated Te in RBCs underwent turnover or was cleared from RBCs during the interval of 4 days. Kidneys had the second highest Te concentration among the tissues measured. Kidney Te concentration increased with repeated administration, suggesting that Te clearance from the kidneys was longer than the 4-day interval. Te was preferably distributed to the lungs and spleen. It is reported that Te was metabolized by methylation, and the resulting TMTe was excreted into urine (Ogra et al., 2007). As a metabolic intermediate, volatile dimethyltelluride could be formed and the lungs seemed to accumulate the volatile metabolite (DeMeio and Jetter, 1948). Since RBCs are destroyed in the spleen, spleen Te concentration may be a result of Te accumulation in RBCs.

$\mathrm{Sb}$ markedly accumulated in RBCs and Sb concentration in RBCs increased with repeated administration (Figs. 1C and D). Sb seemed to more specifically distribute to RBCs than Te. It is well known that there is a large species difference in the tolerance to As toxicity among rodents (Mitchell et al., 2000). The rat is the most tolerant species because it accumulates As in RBCs to reduce distribution in tissues. This suggests that $\mathrm{Sb}$ also shows species difference among rodents. In contrast to Te, however, $\mathrm{Sb}$ did not preferably distribute to the kidneys. The spleen and lung also preferably accumulated $\mathrm{Sb}$ as a result of $\mathrm{Sb}$ accumulation in RBCs.

The organ that most preferably accumulated Ge was the kidneys (Figs. 1E and F). The repeated administration decreased Ge distribution in kidneys compared to the single administration, although the difference was not significant. In contrast to $\mathrm{Te}$ and $\mathrm{Sb}, \mathrm{Ge}$ showed no apparent accumulation in RBCs. 
A. Kobayashi and Y. Ogra

(A)

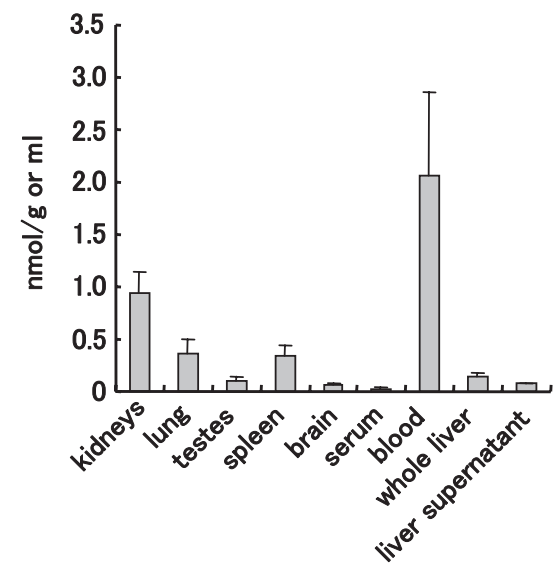

(C)

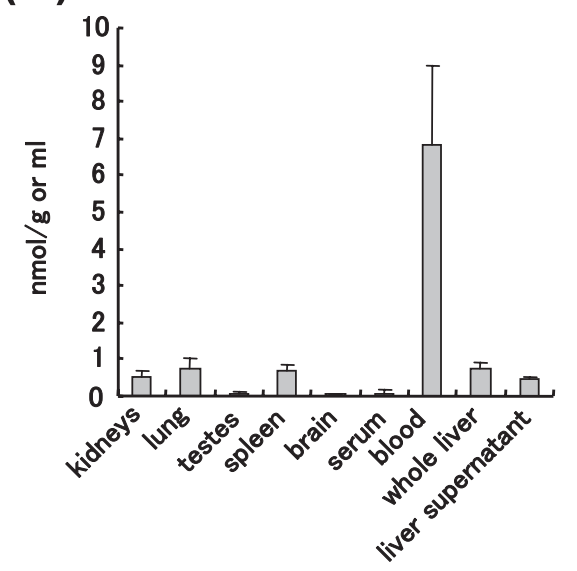

(E)

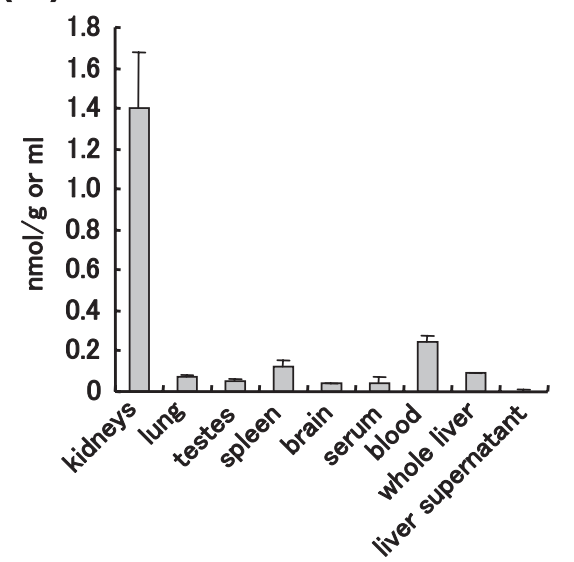

(B)

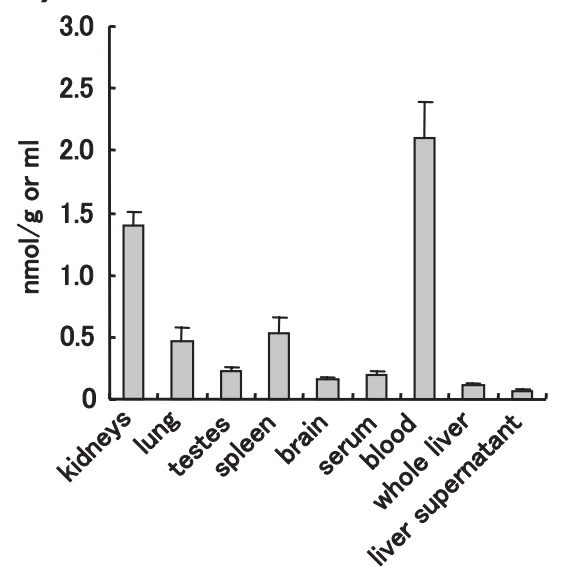

(D)

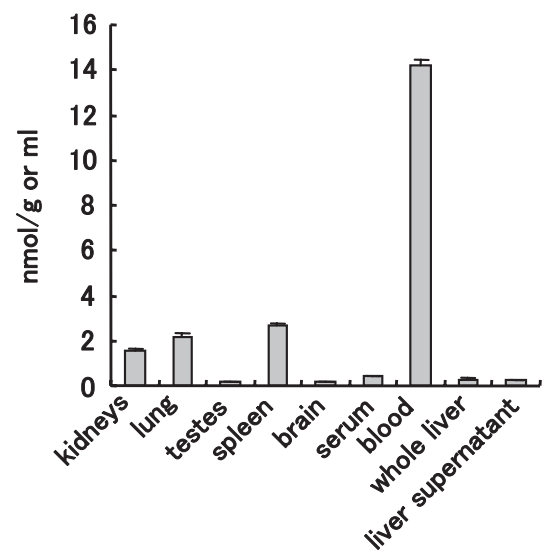

(F)

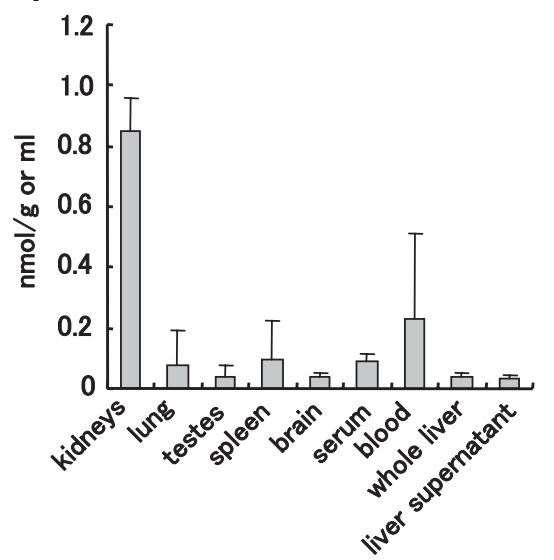

Fig. 1. Concentrations of $\mathrm{Te}, \mathrm{Sb}$ and $\mathrm{Ge}$ in tissues after simultaneous oral administration of the metalloids. Male Wistar rats were singly ( $\mathrm{A}, \mathrm{C}$ and $\mathrm{E})$ or repeatedly $(\mathrm{B}, \mathrm{D}$ and $\mathrm{F})$ administered sodium tellurite, antimony trioxide and germanium dioxide each at a dose of $20 \mu \mathrm{mol} / \mathrm{kg}$ body weight. The concentrations of Te (A and B), Sb (C and D) and Ge (E and F) in tissues were determined by ICP-MS. 


\section{Excretion of metalloids}

Time-dependent changes in urinary excretion after repeated administration of the metalloids were observed (Fig. 2). Te was less and more slowly excreted into urine than the other metalloids; namely, the amount of urinary Te reached a maximum $24 \mathrm{hr}$ after each administration, and was around $0.4 \mu \mathrm{g} / 12 \mathrm{hr}$ (Fig. 2A). The amount of Te excreted into urine increased with the repeated administration, suggesting that the enzyme(s) catalyzing the metabolism of Te was induced, which resulted in the increased urinary excretion of Te. On the other hand, the amounts of urinary $\mathrm{Sb}$ and Ge reached a maximum $12 \mathrm{hr}$ after each administration. Sb excretion continued until 36$48 \mathrm{hr}$ after administration (Fig. 2B), whereas Ge excretion almost reached completion within $12 \mathrm{hr}$ (Fig. 2C). There were no apparent increases in the amounts of $\mathrm{Sb}$ and $\mathrm{Ge}$ excreted into urine in the case of the repeated administration.

The fecal excretion profiles of the three metalloids showed the same tendency, i.e., the amount of fecal metalloid reached a maximum 12-24 hr after administration and returned to basal level $48 \mathrm{hr}$ after administration (Fig. 3).
Of note was that the amount of $\mathrm{Sb}$ in feces was larger than those of the other metalloids, suggesting that $\mathrm{Sb}$ was the least efficiently ingested among the metalloids studied. However, it is required to evaluate whether Se is excreted into bile like As or not in a future study. Compared to $\mathrm{Sb}$, Ge was little excreted into feces and efficiently excreted into urine (Fig. 2), suggesting that Ge was well ingested in the gut and was rapidly excreted into urine. Te was also less excreted into feces than $\mathrm{Sb}$, but was less efficiently excreted into urine than Ge. In addition, Te did not markedly accumulate in the tissues (Fig. 1). In the present study, we did not examine all tissues and thus Te concentrations in other tissues, such as muscle, bone and pancreas, could not be determined. However, it is unlikely that Te accumulated in those tissues. In fact, the clinical manifestations of Te toxicosis patients do not suggest Te accumulation in such tissues. On the other hand, the most common and typical manifestation of Te toxicosis is garlic breath originating from volatile Te compounds. Taken together, Te seemed to be excreted into breath as volatile metabolites, such as dimethylditelluride and dimethyltelluride.
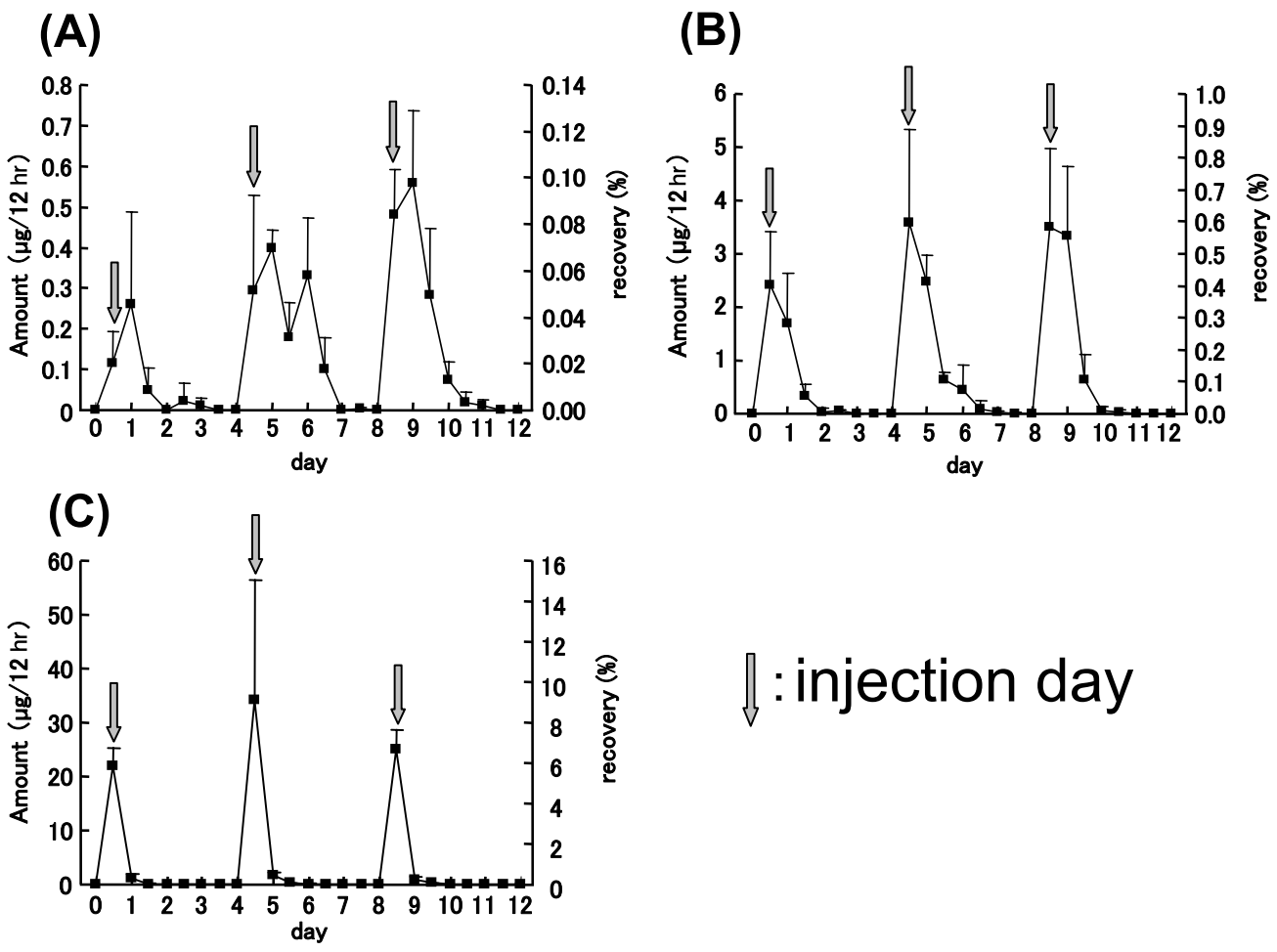

\section{\: injection day}

Fig. 2. Amounts of $\mathrm{Te}, \mathrm{Sb}$ and $\mathrm{Ge}$ in urine after simultaneous oral administration of the metalloids. Male Wistar rats were repeatedly administered sodium tellurite, antimony trioxide and germanium dioxide each at a dose of $20 \mu \mathrm{mol} / \mathrm{kg}$ body weight. The amounts of $\mathrm{Te}(\mathrm{A}), \mathrm{Sb}(\mathrm{B})$ and $\mathrm{Ge}(\mathrm{C})$ in urine sampled over a $12 \mathrm{hr}$ period after administration were determined by ICP-MS. The day the rats were administered the metalloids is indicated by an arrow. 
(A)

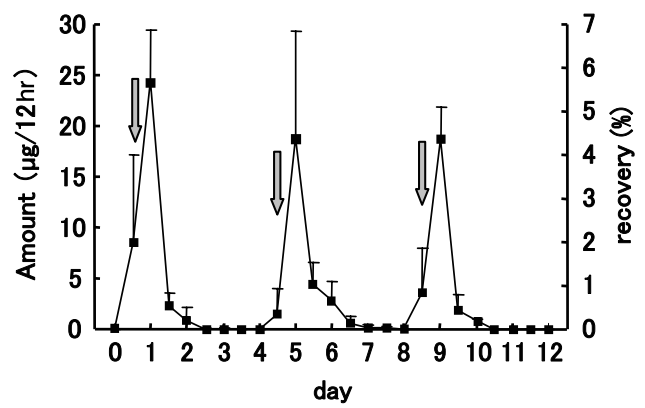

(C)

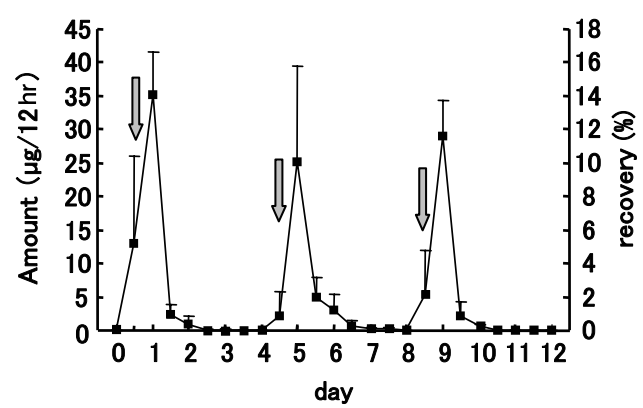

(B)

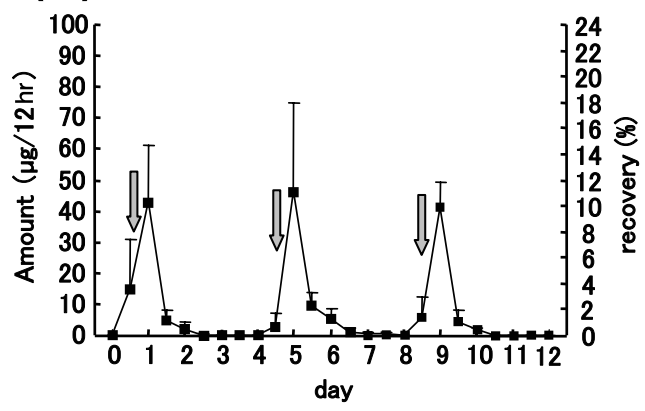

$\sqrt{\|}$ : injection day

Fig. 3. Amounts of Te, $\mathrm{Sb}$ and Ge in feces after simultaneous oral administration of the metalloids. Male Wistar rats were repeatedly administered sodium tellurite, antimony trioxide and germanium dioxide each at a dose of $20 \mu \mathrm{mol} / \mathrm{kg}$ body weight. The amounts of Te (A), Sb (B) and Ge (C) in feces collected over a 12-hr period after administration were determined by ICP-MS. The day the rats were administered the metalloids is indicated by an arrow.

\section{Speciation of metalloids in urine}

Te was eluted as a single peak at the retention time of 19.7 min (Fig. 4A). The chromatographic behavior of the peak was identical to that of the urinary Te metabolite when Te was solely administered in our previous report, i.e., the peak was assigned to TMTe. This indicates that the major urinary Te metabolite is TMTe even when Te is administered simultaneously with $\mathrm{Sb}$ and $\mathrm{Ge}$ at the present dose.

Two major peaks of $\mathrm{Sb}$ were detected in the urine sample (Fig. 4B). The retention time of the large peak matched that of antimonate $(\mathrm{Sb}(\mathrm{V}))$ ), suggesting that the major pathway for $\mathrm{Sb}$ metabolism was oxidation when antimonite (Sb(III)) was administered. On the other hand, the small peak could not be completely assigned yet. It was reported that $\mathrm{Sb}(\mathrm{V})$ was sequestered by some organic acids, such as citric acid, in urine (Hansen and Pergantis, 2006). Indeed, we found that the retention time of the $\mathrm{Sb}(\mathrm{V})$-citrate complex matched that of the small peak on the GS-220HQ column (data not shown). It is well known that arsenite (As(III)) is metabolized by methylation and the major urinary As metabolite of rats is dimethylarsinic acid (DMA (V)) (Suzuki et al., 2002). However, $\mathrm{Sb}$, an element belonging to the same group as As, seems to be slightly or not methylated in the metabolism although trace amounts of $\mathrm{Sb}$ metabolites in urine could not be assigned yet.

A large portion of $\mathrm{Ge}$ in urine was detected as a broad peak at the retention time of $19.1 \mathrm{~min}$ (Fig. 4C). The retention time corresponded to that of germanium oxide, which is the ingested form of Ge. This suggests that Ge is predominantly excreted into urine without being metabolized. The minor peaks were eluted at the retention times of 11-13 min.

To summarize the results of the speciation of urinary metalloids, we found that the metalloids were specifically metabolized via individual pathways. Te was converted into TMTe whose oxidation state was -II, whereas the chemical form of Te ingested was tellurite whose oxidation state was +IV. Thus, tellurite was reduced and trebly methylated to TMTe. Antimonite was simply oxidized to antimonate, while germanium oxide was not metabolized. Methylation takes place in the detoxification of cer- 
tain metalloids, such as Se, Te and As. However, other metalloids, such as $\mathrm{Sb}$ and $\mathrm{Ge}$, are not methylated in the body (Naranmandura et al., 2006; Ogra et al., 2008). In particular, it is remarkable that $\mathrm{Sb}$ is not methylated in rats that have the highest methylation capacity for As among rodents. It is reported that the metabolic intermediates of As, such as monomethylated arsenical (MMA) and dimethylmonothioarsenical (DMMTA), showed specific toxicological effects, such as DNA strand breaking and oxidative stress (Naranmandura et al., 2007; Wang et al., 2007). Although Sb belongs to the same group as As, its metabolic pathway is completely different from that of As. This suggests that $\mathrm{Sb}$ shows different toxicological effects from As.

\section{Speciation of metalloids in RBC lysate}

The peak appearing at the retention time of $18.8 \mathrm{~min}$ showed absorptions at 550 and $280 \mathrm{~nm}$, and the major part of $\mathrm{Fe}$ in the RBC lysate was eluted into the fraction that corresponded to that peak (Fig. 5). Hence, this peak was assigned to hemoglobin $(\mathrm{Hb})$. It is known that As specifically binds to $\mathrm{Hb}$ in rat $\mathrm{RBC}$ lysate, and its chemical form is dimethylated As (DMA) (Lu et al., 2004). In addition, it was reported that Te also accumulated in RBC as dimethylated Te (DMTe) (Ogra et al., 2008). The result obtained in the present study coincided with the previous observations. Sb markedly accumulated in RBCs (Fig. 1) and specifically bound to $\mathrm{Hb}$ (Fig. 5). This indicates that $\mathrm{Hb}$ has a high affinity site for $\mathrm{Sb}$, which resulted in the $\mathrm{Sb}$ accumulation in RBCs. Trace amount of Ge also bound to $\mathrm{Hb}$. To specify the chemical form of $\mathrm{Sb}$ bound to $\mathrm{Hb}$, $\mathrm{Sb}$ was cleaved off from $\mathrm{Hb}$ with $\mathrm{H}_{2} \mathrm{O}_{2}$, and the cleaved $\mathrm{Sb}$ species was subjected to HPLC-ICP-MS. The As compound that bound to $\mathrm{Hb}$ served as positive control and was assigned as DMA. On the other hand, the cleaved $\mathrm{Sb}$ compound showed the same chromatographic behavior as that of antimonate, suggesting that the Sb that specifically bound to $\mathrm{Hb}$ had an inorganic form, not a methylated one (Fig. 6). Therefore, antimonite was directly incorporated into RBCs and specifically bound to $\mathrm{Hb}$ without being metabolized.

\section{Conclusions}

Three exotic metalloids, Te, $\mathrm{Sb}$ and Ge, simultaneously administered to rats were metabolized via a unique pathway. Te was simply methylated. Dimethylated Te accumulated in RBCs and might be excreted into breath. TMTe was the major urinary metabolite of Te. In the case of $\mathrm{Sb}$, methylation was not the major metabolic pathway because almost all $\mathrm{Sb}$ species detected in tissues, urine and feces were of the inorganic form. However, inorgan-

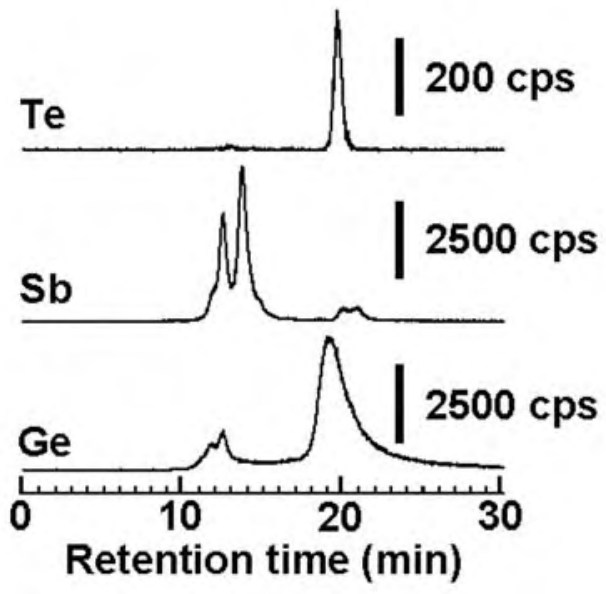

Fig. 4. Elution profiles of $\mathrm{Te}, \mathrm{Sb}$ and $\mathrm{Ge}$ in urine. Urine samples were collected 0-24 hr after administration and a $20 \mu 1$ aliquot of the urine sample was applied to a GS-220HQ column. The column was eluted with 50 $\mathrm{mmol} / 1$ ammonium acetate, $\mathrm{pH} 6.5$, at a flow rate of $0.6 \mathrm{ml} / \mathrm{min}$. The vertical bar indicates the detection level as cps (counts per second).

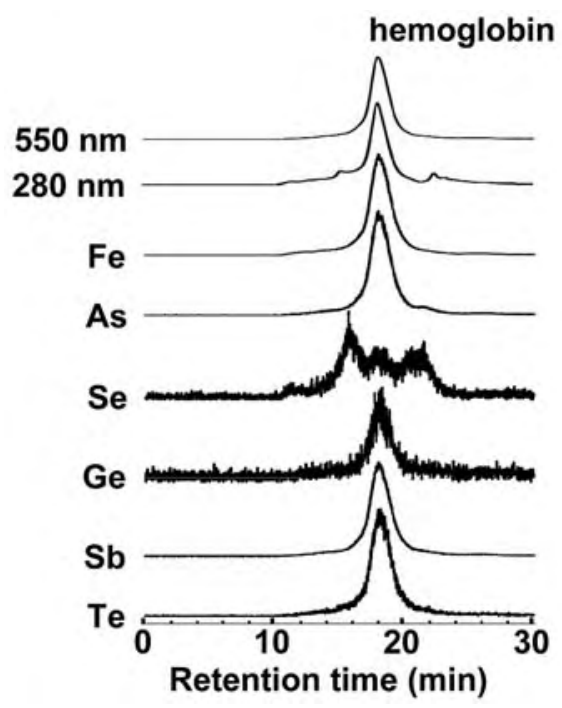

Fig. 5. Elution profiles of elements and UV absorption in RBC lysate. A $200 \mu 1$ aliquot of RBC lysate was applied to a KW803 column. The column was eluted with $50 \mathrm{mmol} / 1$ Tris- $\mathrm{NO}_{3}, \mathrm{pH} 7.4$, at a flow rate of 0.6 $\mathrm{ml} / \mathrm{min}$. UV absorption was monitored at 280 and 550 $\mathrm{nm}$, and the distributions of $\mathrm{Fe}, \mathrm{As}, \mathrm{Se}, \mathrm{Ge}, \mathrm{Sb}$ and $\mathrm{Te}$ were detected at $\mathrm{m} / \mathrm{z} 57,75,82,72,121$ and 125 , respectively. 


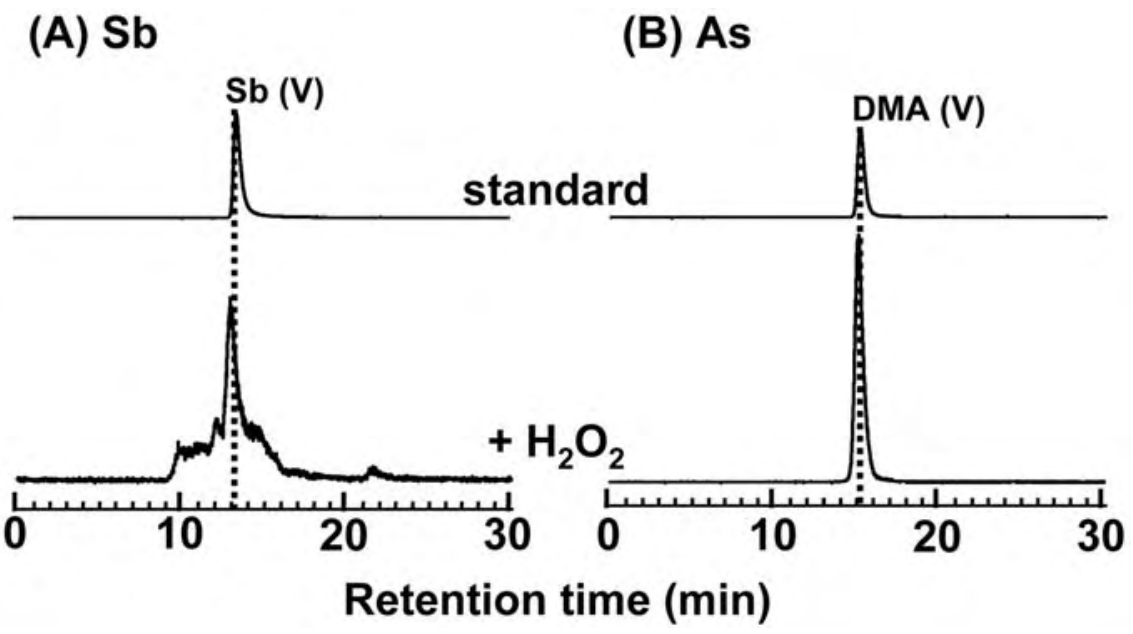

Fig. 6. Elution profiles of $\mathrm{Sb}$ and $\mathrm{As}$ in oxidized RBC lysate. A $20 \mu \mathrm{l}$ aliquot of the oxidized RBC lysate sample was applied to a GS-220HQ column. The column was eluted with $50 \mathrm{mmol} / 1$ ammonium acetate, $\mathrm{pH} 6.5$, at a flow rate of $0.6 \mathrm{ml} / \mathrm{min}$. Elution profiles of $\mathrm{Sb}(\mathrm{A})$ and $\mathrm{As}(\mathrm{B})$ in the sample (lower panels) were compared with those of antimonate and DMA (V) standards (upper panels).

ic $\mathrm{Sb}(\mathrm{III})$ accumulated in RBCs, similarly to dimethylated As. Urinary Sb compounds were $\mathrm{Sb}(\mathrm{V})$, suggesting that the main metabolic pathway of $\mathrm{Sb}$ was oxidation. In the case of Ge, germanium dioxide was more efficiently ingested in the gut than tellurite and antimonite; however, it was efficiently excreted into urine without being metabolized. It is known that the species difference in As toxicity among rodents is highly dependent on the methylation activity (Vahter, 2000). As Sb metabolism seems to be independent of methylation, the species difference in $\mathrm{Sb}$ toxicity may not appear among rodents. Further experiments are needed to resolve these issues.

\section{ACKNOWLEDGMENTS}

The authors would like to acknowledge a Grant-in-Aid from the Ministry of Education, Culture, Sports, Science and Technology, Japan (No. 19390033) and financial support from Agilent Technologies Foundation, USA.

\section{REFERENCES}

DeMeio, R.H. and Jetter, W.W. (1948): Tellurium. The toxicity of ingested tellurium dioxide for rats. J. Ind. Hyg. Toxicol., 30, 5358.

Frei, G.M., Kremer, M., Hanschmann, K.M., Krause, S., Albeck, M., Sredni, B. and Schnierle, B.S. (2008): Antitumour effects in mycosis fungoides of the immunomodulatory, tellurium-based compound, AS101. Br. J. Dermatol., 158, 578-586.
Hansen, H.R. and Pergantis, S.A. (2006): Investigating the formation of an $\mathrm{Sb}(\mathrm{v})$-citrate complex by HPLC-ICP-MS and HPLCES-MS(/MS). J. Anal. At. Spectrom., 21, 1240-1248.

Haraguchi, H. (2004): Metallomics as integrated biometal science. J. Anal. At. Spectrom., 19, 5-14.

Hayakawa, T., Kobayashi, Y., Cui, X. and Hirano, S. (2005): A new metabolic pathway of arsenite: Arsenic-glutathione complexes are substrates for human arsenic methyltransferase Cyt19. Arch. Toxicol., 79, 183-191.

Hirayama, C., Suzuki, H., Ito, M., Okumura, M. and Oda, T. (2003): Propagermanium: A nonspecific immune modulator for chronic hepatitis B. J. Gastroenterol., 38, 525-532.

Krachler, M. and Emons, H. (2001): Urinary antimony speciation by HPLC-ICP-MS. J. Anal. At. Spectrom., 16, 20-25.

Lu, M., Wang, H., Li, X.F., Lu, X., Cullen, W.R., Arnold, L.L., Cohen, S.M. and Le, X.C. (2004): Evidence of hemoglobin binding to arsenic as a basis for the accumulation of arsenic in rat blood. Chem. Res. Toxicol., 17, 1733-1742.

McCallum, R.I. (2005): Occupational exposure to antimony compounds. J. Environ. Monit., 7, 1245-1250.

Mitchell, R.D., Ayala-Fierro, F. and Carter, D.E. (2000): Systemic indicators of inorganic arsenic toxicity in four animal species. J. Toxicol. Environ. Health A, 59, 119-134.

Naranmandura, H., Suzuki, N. and Suzuki, K.T. (2006): Trivalent arsenicals are bound to proteins during reductive methylation. Chem. Res. Toxicol., 19, 1010-1018.

Naranmandura, H., Ibata, K. and Suzuki, K.T. (2007): Toxicity of dimethylmonothioarsinic acid toward human epidermoid carcinoma A431 cells. Chem. Res. Toxicol., 20, 1120-1125.

Ogra, Y., Ishiwata, K., Takayama, H., Aimi, N. and Suzuki, K.T. (2002): Identification of a novel selenium metabolite, Se-methyl$\mathrm{N}$-acetylselenohexosamine, in rat urine by high-performance liquid chromatography-inductively coupled plasma mass spectrometry and -electrospray ionization tandem mass spectrometry. 
Metabolism of $\mathrm{Te}, \mathrm{Sb}$ and $\mathrm{Ge}$ in rats

J. Chromatogr. B: Anal. Technol. Biomed. Life Sci., 767, 301312.

Ogra, Y., Kobayashi, R., Ishiwata, K. and Suzuki, K.T. (2007): Identification of urinary tellurium metabolite in rats administered sodium tellurite. J. Anal. At. Spectrom., 22, 153-157.

Ogra, Y., Kobayashi, R., Ishiwata, K. and Suzuki, K.T. (2008): Comparison of distribution and metabolism between tellurium and selenium in rats. J. Inorg. Biochem., 102, 1507-1513.

Poon, R., Chu, I., Lecavalier, P., Valli, V.E., Foster, W., Gupta, S. and Thomas, B. (1998): Effects of antimony on rats following 90-day exposure via drinking water. Food Chem. Toxicol., 36, 21-35.

Santos, D.O., Coutinho, C.E.R., Madeira, M.F., Bottino, C.G., Vieira, R.T., Nascimento, S.B., Bernardino, A., Bourguignon, S.C., Corte-Real, S., Pinho, R.T., Rodrigues, C.R. and Castro, H.C. (2008): Leishmaniasis treatment -- A challenge that remains: A review. Parasitol. Res., 103, 1-10.

Sharma, P., Perez, D., Cabrera, A., Rosas, N. and Arias, J.L. (2008): Perspectives of antimony compounds in oncology. Acta Pharmacol. Sin., 29, 881-890.

Suzuki, K.T., Mandal, B.K. and Ogra, Y. (2002): Speciation of arsenic in body fluids. Talanta, 58, 111-119.

Szpunar, J. (2005): Advances in analytical methodology for bioinor- ganic speciation analysis: Metallomics, metalloproteomics and heteroatom-tagged proteomics and metabolomics. Analyst, 130, 442-465.

Taylor, A. (1996): Biochemistry of tellurium. Biol. Trace Elem. Res., 55, 231-239.

Vahter, M. (2000): Genetic polymorphism in the biotransformation of inorganic arsenic and its role in toxicity. Toxicol. Lett., 112113, 209-217.

Vanholder, R., Cornelis, R., Dhondt, A. and Lameire, N. (2002): The role of trace elements in uraemic toxicity. Nephrol. Dial. Transplant., 17, 2-8.

Wang, T.C., Jan, K.Y., Wang, A.S.S. and Gurr, J.R. (2007): Trivalent arsenicals induce lipid peroxidation, protein carbonylation, and oxidative DNA damage in human urothelial cells. Mutat. Res. Fundam. Mol. Mech. Mutagen., 615, 75-86.

Yamada, N., Kojima, R., Uno, M., Akiyama, T., Kitaura, H., Narumi, K. and Nishiuchi, K. (2002): Phase change material for use in rewritable dual-layer optical disk. Proc. SPIE-Int. Soc. Opt. Eng., 4342, 55-63.

Yamasoba, T., Someya, S., Yamada, C., Weindruch, R., Prolla, T.A. and Tanokura, M. (2007): Role of mitochondrial dysfunction and mitochondrial DNA mutations in age-related hearing loss. Hear. Res., 226, 185-193. 